\title{
Quantification of Amino Acid Neurotransmitters in Cerebrospinal Fluid of Patients with Neurocysticercosis
}

\author{
José Augusto Camargo* and Paulo Henrique Ferreira Bertolucci*
}

\begin{abstract}
Departamento De Neurologia E Neurocirurgia Da Universidade Federal De São Paulo/Hospital São Paulo, São Paulo, Brasil
\end{abstract}

\begin{abstract}
Background: Neurocysticercosis is a parasitic disease that affects the central nervous system. Its main clinical manifestations are epileptic seizures. The objective of this study was to investigate the correlation between neurotransmitter concentrations in cerebrospinal fluid (CSF) and the different evolutive forms of neurocysticercosis with or without seizures.

Methods: Neurotransmitter concentrations (Aspartate, Glutamate, GABA, Glutamine, Glycine, Taurine) were determined in CSF samples from 42 patients with neurocysticercosis divided into patients with the active cystic form $(\mathrm{n}=24,12$ with and 12 without seizures) and patients with calcified form ( $\mathrm{n}=18,12$ with and 6 without seizures), and a control group consisting of 59 healthy subjects.
\end{abstract}

Results: Alterations in amino acid concentration were observed in all patients with neurocysticercosis.

Conclusion: We conclude that disturbances in amino acid metabolism accompany the presentation of neurocysticercosis. Replacement of the terms inactive cyst by reactive inactive cyst and calcification by reactive calcification is suggested.

Keywords: Neurocysticercosis, neurotransmitters, neuronal damage.

\section{INTRODUCTION}

Neurocysticercosis (NCC) is a term used to define involvement of the central nervous system (CNS) by the larval form of the tapeworm Taenia solium. It is the most common cause of adult-acquired epilepsy worldwide and one of the most frequent parasitic infections associated with chronic morbidity in the United States. Despite its importance, worldwide morbidity due to NCC is underappreciated and research is underfunded, and therefore researchers are unable to capitalize on recent advances that hold great promise to prevent millions of cases of epilepsy and to effectively treat viable brain infections. Mature cysticerci remain viable in the brain parenchyma for 3 to 6 years, a period during which the cysticercus modulates the immune response of the host in such a way as to prevent its destruction and to permit the peaceful coexistence of the parasite and host [1-3]. In contrast, during the degenerative phase the cysticercus is unable to suppress the action of the host immune system and is destructive. The symptoms observed during this phase are generally associated with the inflammatory response and clinical manifestations, but also depend on the number, morphological type and localization of the cysts [4-6].

The time to symptom development in the series by Dixon and colleagues [7] refuted the intuitive interpretation that the entry of the parasites into the brain was responsible for the symptoms.

*Address correspondence to these authors at the Av Juscelino Kubitschek de Oliveira 753 / sala 11, 18035-060 Sorocaba SP - Brasil;

E-mail: jacneuro@hotmail.com
Clinical manifestations of cysticercosis include seizures, intracranial hypertension, meningitis and dementia. Many studies suggest that, as along as the cyst continues to be viable, the patient remains asymptomatic [8, 9].

The three evolutive stages of the cysticercus, i.e., viable, degenerative and calcified, can be identified by neuroimaging methods. In contrast, analysis of cerebrospinal fluid (CSF) provides important information about the inflammatory response that occurs in NCC, permitting a better understanding of the interaction between the parasite and host [10-12]. It seems increasingly more inadequate to classify NCC lesions into active or inactive based only on imaging criteria, since inactive forms detected by imaging exams might present important clinical and CSF manifestations [1]. As mentioned earlier, the main clinical manifestations of NCC are epileptic seizures. These manifestations are directly associated with altered synaptic transmission, which results from changes in the concentrations of amino acid neurotransmitters in the CNS. Epileptogenesis in patients with calcified neurocysticercosis has been a subject of debate. While calcifications have been considered inert lesions, recent data suggest that calcified cysticerci are not clinically inactive nor pathologically inert lesions, as they may cause recurrent seizures and cognitive decline when parasitic antigens trapped in the calcium matrix are exposed to the host immune system due to a process of calcification remodeling [13].

Synaptic transmission refers to the propagation of nerve impulses from one cell to another. This occurs in specialized cellular structures, called synapses, where the axon of a presynaptic neuron connects at some site to a postsynaptic neuron. Nerve impulses are transmitted at the synapses through 
the release of chemical substances, the neurotransmitters. Some studies have demonstrated the involvement of monoamines and amino acids in epileptic phenomena. In this respect, alterations in the metabolism of catecholamines, serotonin and the amino acids aspartate, glutamate and gammaamino butyric acid (GABA) have been reported. In the brain, the synthesis and metabolism of aspartate and glutamate occur in neurons and astrocytes. Most of the glutamate released at presynaptic terminals is taken up by glial cells and converted into glutamine. Glutamine is then transported to the neuron and the cycle is completed by its conversion into glutamate and aspartate. GABA is synthesized from glutamate. Many studies indicate GABA as the main inhibitory neurotransmitter in the brain, which increases the synchronization of interneurons, generating an inhibitory response. Like GABA, glycine and taurine also act by inhibiting action potential firing in neurons. Glutamate and aspartate exert an excitatory action by acting as endogenous ligands for receptors present in the mammalian CNS, determining excitatory responses in neuronal cells $[14,15]$.

Taurine is one of the most abundant amino acids in the CNS and is synthesized from cysteine by cysteine sulfinic acid decarboxylase [16].

Glutamate is the main excitatory amino acid and excessive activation of glutamate receptors is called excitotoxicity, an event that is observed in various neuropathological states such as epileptic seizures, ischemia, anoxia, hypoglycemia, and inflammation associated with viral infection [17].

Seizures are observed in many patients with NCC who present lesions classified as inactive or calcified according to imaging criteria, whereas others do not present any seizure episodes throughout life. The same is observed for patients with lesions classified as active, patients with cysts without a halo of perilesional edema and patients who do not show any signs of an inflammatory reaction upon computed tomography (CT) or nuclear magnetic resonance (MR) imaging and $\mathrm{CSF}$ analysis. The causes of this observation are still uncertain but might be related to alterations in neurotransmitter concentrations in the nervous system. Since neurotransmitter levels in CSF reflect central changes, their quantification may provide relevant data regarding the genesis of clinical manifestations of many diseases, including NCC.

The objective of this study was to investigate the correlation between neurotransmitter concentrations in CSF and the different evolutive forms of neurocysticercosis with or without epileptic seizures.

\section{MATERIALS AND METHODS}

\subsection{Patients}

Patients with NCC were diagnosed based on a combination of imaging findings (cranial CT and MR), CSF alterations including an immunoenzymatic reaction to NCC, epidemiological criteria, and clinical findings according to the criteria of Del Brutto et al. (2001) [4].
A total of 101 patients of both genders, ranging in age from 19 to 59 years, were studied.

Using the classification suggested by Carpio et al. (1994) [18], with modifications, the patients were subdivided into five groups according to the evolutive stage of the cysticercus in the absence of signs of an inflammatory reaction in the imaging exam and CSF analysis: 1) patients with one or more active or viable cysts in the absence of signs of an inflammatory reaction without epileptic seizures $(n=12)$; 2) patients with one or more active or viable cysts in the absence of signs of an inflammatory reaction with seizures $(\mathrm{n}=12) ; 3)$ patients with one or more inactive of calcified cysts in the absence of signs of an inflammatory reaction without seizures $(n=6)$; 4$)$ patients with one or more inactive or calcified cysts in the absence of an inflammatory reaction with seizures $(n=12) ; 5)$ control group $(n=59)$ consisting of patients without neurological or systemic alterations who were submitted to lumbar puncture for spinal anesthesia due to other reasons, such as minor orthopedic surgery, surgery for inguinal hernia correction, etc. All patients with seizures who presented hydrocephalus and febrile signs and whose last seizure episode occurred within a period of less than 4 days and more than 30 days were excluded from the study.

Patients included in the study who belonged to the group with calcified cysts were subjected to CSF puncture for the investigation of possible reinfestation with cysticerci, associated with symptoms such as limb paresthesia and dizziness, vertigo and tinnitus. After clinical investigation, these symptoms were diagnosed as part of osteoarticular diseases of the spine and vestibular system disorders, respectively .CSF punctures were performed within a period of 4 to 30 days after the last seizure episode in patients of the groups presenting epileptic seizures.

All subjects were subjected to CT scan or brain MR. All subjects were submitted to lumbar puncture and CSF cytomorphological examination and are not taking antiepileptic drugs.

The project was approved by the Ethics Committee of UNIFESP (process 0857/05).

\subsection{Collection of Cerebrospinal Fluid}

CSF samples were collected by the responsible physician after the patient had signed a free informed consent form according to the guidelines of the Brazilian Academy of Neurology. Samples containing red blood cells due to traumatic lumbar puncture were discarded. The material collected was transported on ice, divided into aliquots and frozen at $-70^{\circ} \mathrm{C}$ until the time for processing at the laboratory.

\subsection{Sample Preparation for HPLC Analysis and Chro- matographic Conditions}

The CSF samples were thawed and $3 \mathrm{~mL}$ were added to $26 \mu \mathrm{L} 1 \mathrm{mM}$ homoserine, used as the internal standard, and $52 \mu \mathrm{L}$ 11.6 M perchloric acid. The samples were kept on ice 


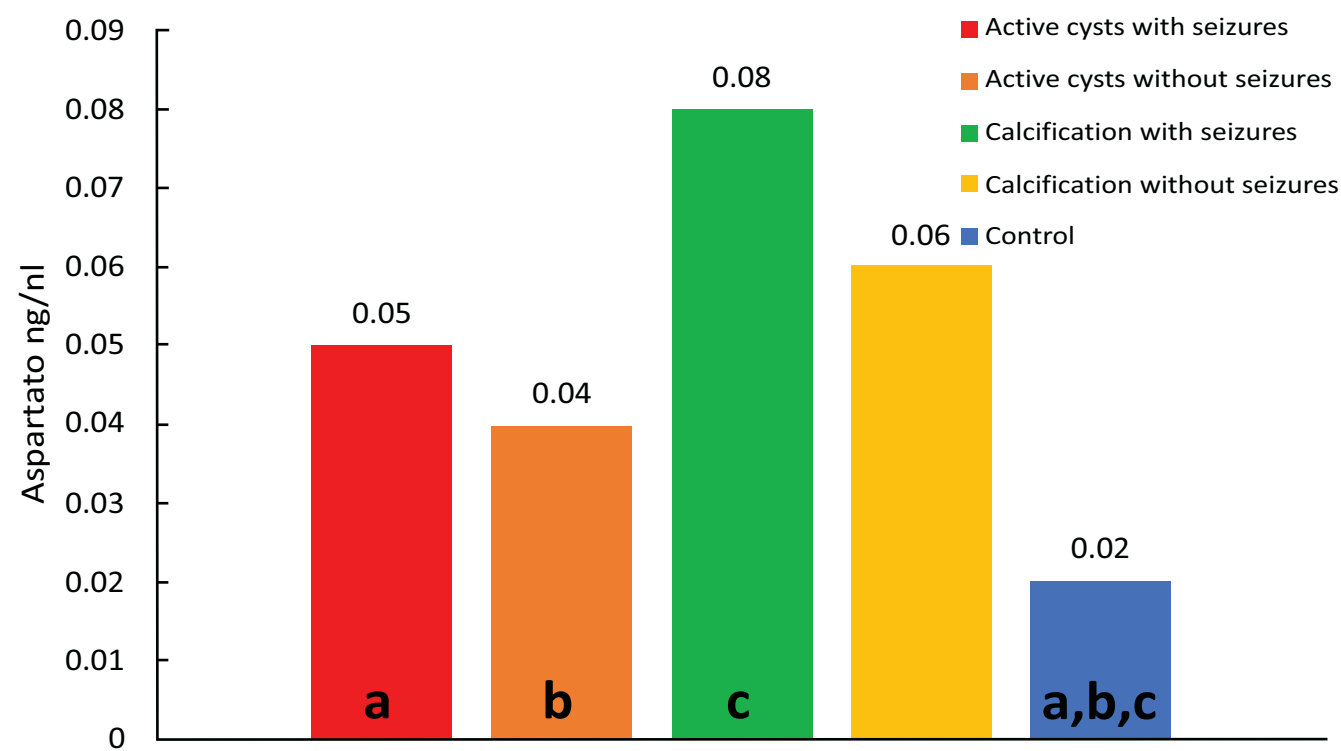

Fig. (1). Median values of Aspartate; diferences: (a) $p<0.001$; (b) $p<0.001$; (c) $p<0.001$.

for $60 \mathrm{~min}$, centrifuged at $10,000 \mathrm{~g}$ for $30 \mathrm{~min}$, and injected into the liquid chromatograph for separation of amino acids. The mobile phase consisted of phosphate buffer, $\mathrm{pH}$ 5.5, and a gradient of methanol. A fluorescence detector was used for the separation of aspartate, glutamate, glycine, taurine, glutamine, and GABA. The concentrations of the amino acids were determined based on the peak height of each neurotransmitter in the sample identified by comparison with a standard run containing known concentrations of each neurotransmitter. The neurotransmitter concentration was calculated using the following formula: $[\mathrm{NT}]=$ peak height $\mathrm{sam}_{\text {- }}$ ple $/$ homoserine/peak height standard $_{\text {homoserine }} \mathrm{x}$ dilution factor of the sample.

\subsection{Statistical Analysis}

Kruskal-Wallis test was used for comparison of amino acids between groups and the Mann-Whitney test adjusted by Bonferroni correction. The decisions were based on a level of significance of $5 \%$ for comparison between groups.

\section{RESULTS}

Comparison of amino acid neurotransmitter concentrations between groups showed the following results.

\subsection{Aspartate}

Comparison of control versus viable cysts with seizures $(\mathrm{p}=0.014)$ showed lower median aspartate concentrations in the control group. Higher levels were observed for the group with viables cysts and seizures when compared to the other groups, with a wider dispersion of values above than below the median. The smallest dispersion was observed for the control group. A higher dispersion of the results was also observed for the calcification group with seizures. (Fig. 1).

\subsection{Glutamate}

Comparison of control versus viable cysts with seizures $(\mathrm{p}=0.011)$ showed lower median glutamate concentrations in the control group. Fig. (2) shows a wider dispersion of the results for the group of viable cysts with seizures compared to the other groups. (Fig. 2).

\subsection{Glutamine}

Comparison of control versus viable cysts without seizures $(\mathrm{p}=0.004)$ showed lower median glutamine concentrations in the control group. Comparison of control versus calcifications without seizures $(\mathrm{p}=0.006)$ showed lower mean and median levels in the control group.

\subsection{GABA}

Comparison of control versus viable cysts with seizures $(\mathrm{p}=0.037)$ showed lower median GABA concentrations in the control group. Comparison of control versus calcifications with seizures $(\mathrm{p}=0.011)$ showed lower median levels in the control group. Comparison of control versus calcifications without seizures $(\mathrm{p}=0.001)$ showed lower median GABA concentrations in the control group (Fig. 3).

The samples were classified into active cysts, calcified cysts and controls, and the groups were then subdivided into patients with and without seizures. All patients were submitted to CT or cranial MR to be included in the study. CT or MR imaging and CSF analysis, including cytomorphological examination, protein quantification and electrophoresis, and glucose and chloride measurement, revealed no signs of an inflammatory reaction in any of the patients. All patients had multiple intraparenchymatous cysts. Images showing 6 to 100 cysts were classified as multiple cysts, corresponding to moderate infestation according to Garcia et al. (2002) [14]. 


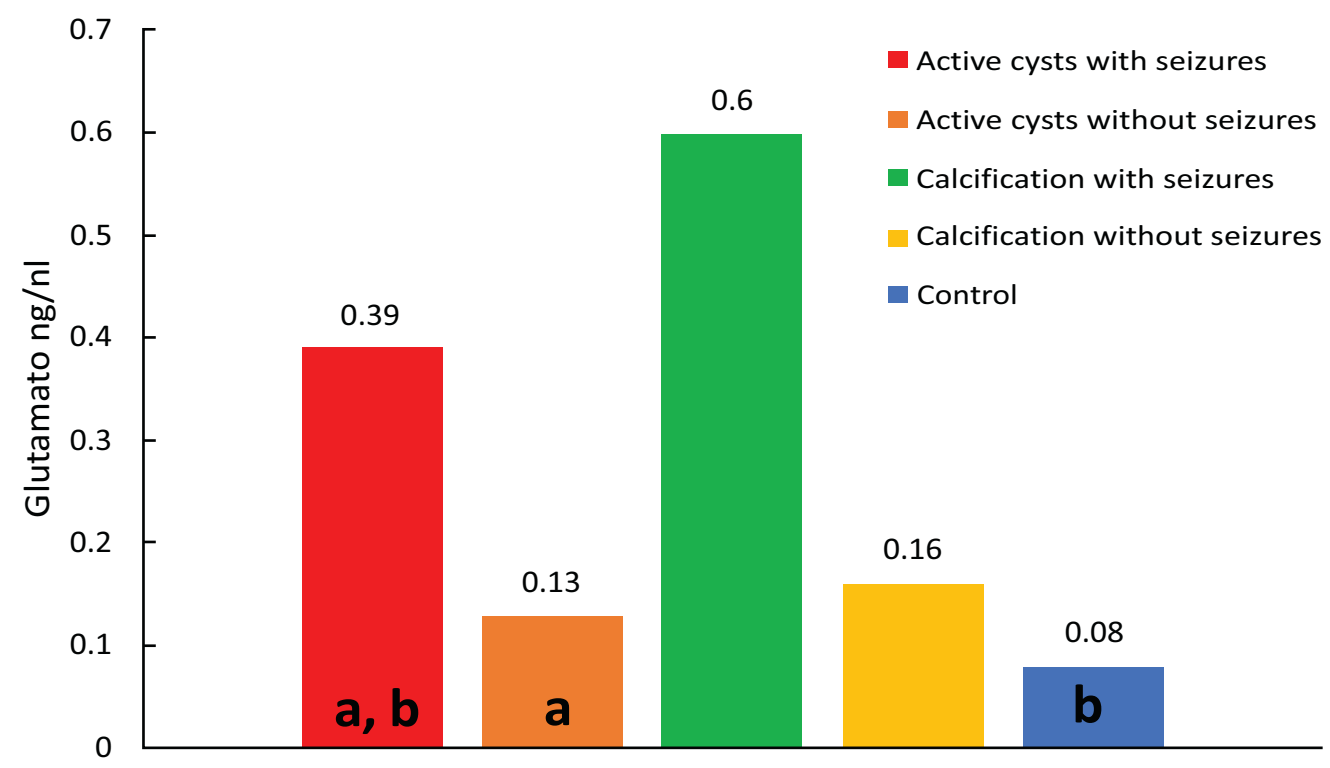

Fig. (2). Median values of glutamate; differences: (a) $p=0.002$; (b) $p<0.001$.

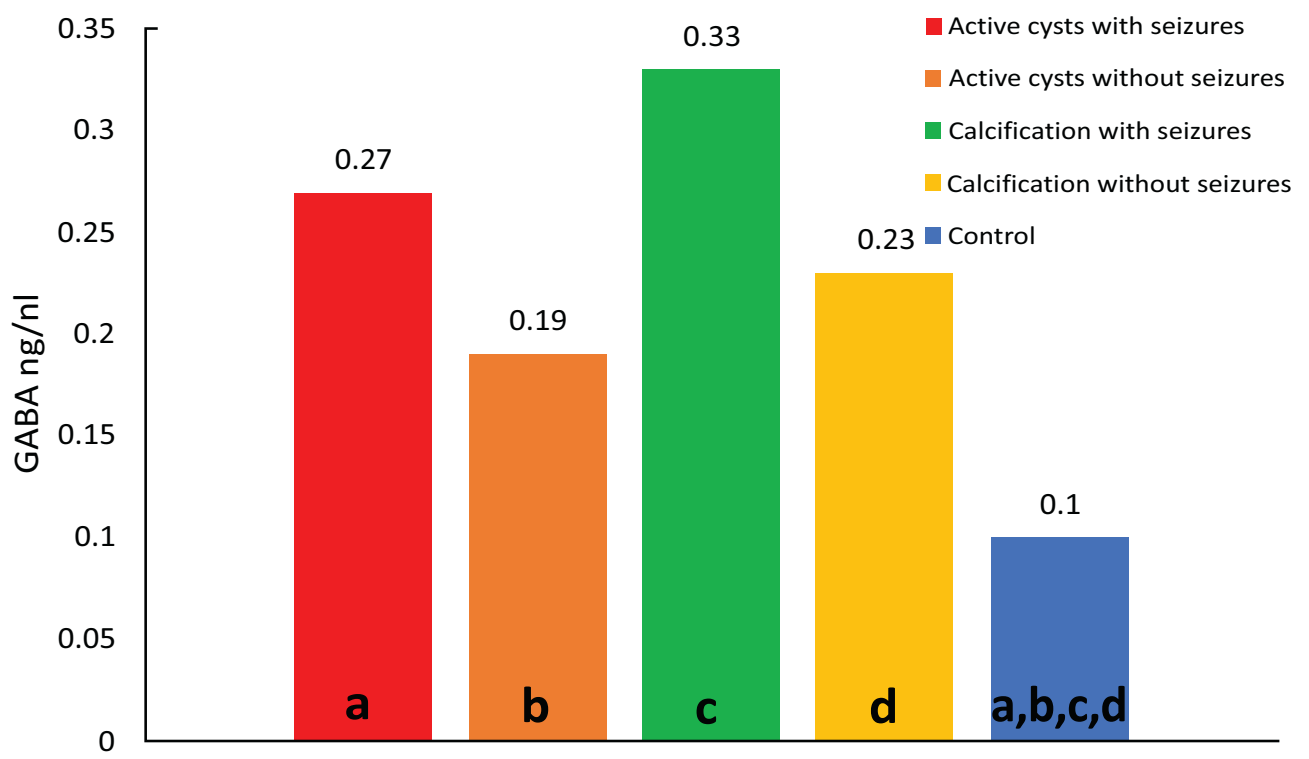

Fig. (3). Median values of GABA; differences: (a) $p=0.001$; (b) $p<0.001$; (c) $p<0.001$; (d) $p<0.001$.

\section{DISCUSSION}

Many questions regarding NCC still need to be elucidated. One of them is the manifestation of seizures in patients who present calcified or viable cysts in the absence of inflammatory signs upon imaging exams (CT and MR) and CSF analysis. This question led us to speculate whether factors exist in the CSF of these patients that predispose to the occurrence of seizures. We therefore studied the CSF concentrations of amino acid neurotransmitters in patients with active and calcified NCC with or without seizures who presented no signs of inflammatory activity, in order to evaluate the occurrence of a possible imbalance between excitatory neurotransmitters, mainly represented by the amino acids aspartate and glutamate, and inhibitory neurotransmitters represented by GABA, taurine, glycine and glutamine.
The present study showed a significant increase in the concentration of aspartate, glutamate and GABA in CSF of patients with viable cysts, and of GABA in CSF of patients with calcified cysts. In both cases the patients presented epileptic seizures. The finding of elevated aspartate and glutamate levels in CSF of patients with viable cysts who presented seizures suggests that these amino acids may predispose to the occurrence of seizures.

These data are indicative that other factors in addition to an increase in excitatory amino acids modulate the excitability of the brain and predispose to epileptic seizures.

Some investigators reported an association between altered amino acid concentrations and predisposition to seizures. Hajek et al. (1997) [19] quantified neurotransmitters in CSF samples from two epileptic patients with arachnoid 
Table 1. Pair of groups.

\begin{tabular}{|c|c|c|c|c|c|c|c|c|c|c|}
\hline \multirow{2}{*}{ Variable } & \multicolumn{10}{|c|}{ Pair of Groups } \\
\hline & $A \times B$ & $A \times C$ & $A \times D$ & A $\times$ CTRL & B $\times$ C & B $\times$ D & B x CTRL & C x D & C x CTRL & D x CTRL \\
\hline ASP & 0.413 & 0.925 & 0.126 & $<0.001$ & 0.573 & 0.167 & $<0.001$ & 0.252 & $<0.001$ & 0.291 \\
\hline GLU & 0.002 & 0.579 & 0.019 & $<0.001$ & 0.090 & 0.299 & 0.622 & 0.450 & 0.012 & 0.047 \\
\hline GLN & 0.355 & 0.667 & 0.160 & $<0.001$ & 0.196 & 0.001 & $<0.001$ & 0.615 & $<0.001$ & $<0.001$ \\
\hline GLI & 0.931 & 0.229 & 0.962 & $<0.001$ & 0.184 & 0.814 & 0.001 & 0.290 & $<0.001$ & 0.002 \\
\hline TAU & 0.009 & 0.947 & 0.288 & 0.005 & 0.016 & 0.005 & 0.853 & 0.314 & 0.005 & 0.003 \\
\hline GABA & 0.203 & 0.324 & 0.606 & $<0.001$ & 0.031 & 0.100 & $<0.001$ & 0.448 & $<0.001$ & $<0.001$ \\
\hline
\end{tabular}

(Bonferroni $\alpha=0.005116$ )

A - Active cysts with seizures; B - Active cysts without seizures; C - Calcification with seizures; D - Calcification without seizures; CTRL - control.

brain cysts detected by MR, who were treated surgically. High levels of the excitatory amino acids aspartate and glutamate were observed in the cystic fluid, whereas there was only a mild increase of glutamate in the epileptogenic brain area adjacent to the cyst in one of the patients. No increase in amino acids was observed in non-epileptogenic brain regions, findings that led the authors to suggest that drug resistance was associated with the presence of intracystic amino acids. Wieser et al. (2003) [20] studied the CSF concentration of amino acids in 23 patients with arachnoid brain cysts submitted to surgery and observed that only three presented elevated levels of aspartate and/or glutamate in the cysts. Only two of these patients presented seizures before surgery. The authors concluded that there is no evidence for a correlation between the presence of excitatory amino acids in brain cysts and the occurrence of seizures. However, elevated levels of adenosine were observed in cystic fluid, a neuromodulator known to have anticonvulsant properties and that might act as a protective factor against seizures. Bestetti (1994) [21] observed no association between the results of CSF analysis of amino acid neurotransmitters and the interval between puncture and the last seizure episode.

Analysis of amino acids in CSF from patients with calcified cysts who did not present seizures revealed significantly elevated glutamine and GABA concentrations. The concomitant increase in GABA and glutamine levels indicates that the glutamine-glutamate-GABA system is activated, protecting the patient against the occurrence of seizures. Glutamine is part of the glutamine-glutamate-GABA cycle and, at the same time, is responsible for the disintoxication of ammonia present in tissue. This amino acid crosses the blood-brain barrier and is taken up by neurons, where it is converted into glutamate by glutaminase. Glutamate, in turn, is converted into GABA by glutamic acid decarboxylase. An increase in GABA concentration was also detected in CSF of patients with viable and calcified cysts who presented seizures, suggesting that other factors might modulate the occurrence of seizures. A higher activity of the glutamine-glutamateGABA cycle seems to be present in patients with viable cysts who present seizures, but this increased activity does not seem to be sufficient to metabolize all glutamate which is found to be elevated in the CSF of these patients (Table 1). In the group of patients with calcified cysts who presented seizures, glutamate and glutamine levels seem to be normal, whereas GABA is elevated, a finding suggesting that disturbances in other neuromodulator systems might contribute to the occurrence of seizures. In this respect, adenosine has been reported to be involved in the modulation of seizures [16].

The present study demonstrated an increase of CSF glutamine concentration in the patients of all groups; however, this increase was significant only in the groups with viable and calcified cysts who did not present seizures, a finding suggesting an efficient anticonvulsant system for the removal of ammonia and glutamate from the extracellular medium, accompanied by the synthesis of GABA. The elevated glutamate levels observed in CSF of patients with viable cysts who presented seizures indicate that an increase in glutamine exerts an important anticonvulsant function. The findings show that this system is increased in both the viable and calcified forms of the disease. However, the imbalance favoring an increase in glutamate and aspartate in the viable form may contribute to the occurrence of seizures. The fact that GABA, but not glutamate or aspartate levels, were elevated in CSF of patients with calcified cysts who presented seizures reflects an inhibitory mechanism of GABA that compensates for the epileptiform process installed.

Although elevated taurine levels were observed in all groups, the difference was not statistically significant. The same was observed for glycine.

The present results suggest a reflexion about the terms inactive cyst proposed by Sotelo et al. (1985) [22] and Carpio et al. (1994) [18] and calcification. We often observe that physicians and healthcare professionals who are not familiar with NCC falsely assume that the disease is resolved, harmless or cured and will not result in further damage when analyzing the reports or results of imaging exams (CT and MR) containing the expressions inactive cyst or calcification. However, as seen in the present study and others even when 
calcified, the cyst can cause local reactions in the host. Within this context, we believe that it is more adequate to replace the terms inactive cyst with reactive inactive cyst $[23,24]$ and calcification with "reactive calcification" clarifying that, even when calcified, the cyst can provoke damaging host tissue reactions.

\section{CONCLUSION}

The increase of glutamate and aspartate levels observed in CSF of patients with viable cysts who present seizures indicates the participation of these amino acids in the genesis of seizures. The elevated GABA levels found in CSF of patients with viable and calcified cysts who present seizures suggest an inhibitory compensatory mechanism which is increased but inefficient, probably because of the absence of other neuromodulator systems such as the adenosine system. The glutamine-glutamate-GABA cycle is increased in the brain of patients with viable and calcified cysts who do not present seizures, representing an important protection mechanism.

Despite the lack of inflammatory signs upon imaging exams and CSF analysis, a dynamic metabolic and inflammatory reaction might be present in the brain parenchyme of patients with NCC. The present results emphasize the importance to consider NCC as a progressive and active disease, even when the cysts are in the calcified stage. Replacement of the term "inactive cyst" by "reactive inactive cyst" and calcification by "reactive calcification" is suggested.

\section{CONFLICT OF INTEREST}

The authors confirm that this article content has no conflict of interest.

\section{ACKNOWLEDGEMENTS}

We are grateful to FADA, CNPQ and FAPESP for financial support.

\section{REFERENCES}

[1] Takayanagui OM, Leite JP. Neurocisticercose. Rev Soc Bras Med Trop 2001; 34: 283-90.

[2] Grewal JS, Kaur S, Bhatti G, et al. Cellular immune responses in human neurocysticercosis. Parasitol Res 2000; 86: 500-3.

[3] Nash TE, Mahanty S, Garcia HH. Neurocysticercosis-more than a neglected disease. PLoS Negl Trop Dis 2013; 7(4): e1964. doi: 10.1371/journal.pntd.0001964.

[4] Del Brutto OH, Rajshekhar V, White AC Jr, et al. Proposed diagnostic criteria for neurocysticercosis. Neurology 2001; 57: 177-83.
[5] Patel R, Jha S, Yadav RK. Pleomorphism of the clinical manifestations of neurocysticercosis. Trans R Soc Trop Med Hyg 2006; 100: 134-41.

[6] Takayanagui OM, Odashima NS. Clinical aspects of neurocysticercosis. Parasitol Int 2006; 55: S111-5.

[7] Dixon HBF, Hargreaves WH. Cysticercosis (Taenia solium): a further ten years' clinical study, covering 284 cases. Q J Med 1944; 13: 107-21.

[8] Ostrosky-Zeichner L, Estanol B. Immunopathogenesis of neurocysticercosis: is damage mediated by the host immune response? Int $\mathbf{J}$ Parasitol 1999; 29: 649-50.

[9] White Jr AC. Neurocysticercosis: updates on epidemiology, pathogenesis, diagnosis, and management. Annu Rev Med 2000; 51: 187-206.

[10] Machado LR, Livramento JA, Vaz AJ, et al. IgG intrathecal synthesis and specific antibody index in patients with neurocysticercosis. Arq Neuropsiquiatr 2002; 60(2-B): 395-9.

[11] García HH, Gonzalez AE, Rodriguez S, et al. Neurocysticercosis: unraveling the nature of the single cysticercal granuloma. Neurology 2010; 75(7): 654-8.

[12] Abraham R, Livramento JA, Leite Cda C, Pardini AX, Vaz AJ, Machado Ldos R. Neurocysticercosis: Relationship between taenia antigen levels in CSF and MRI. Arq Neuropsiquiatr 2010; 68: 7-11.

[13] Rodrigues CL, de Andrade DC, Livramento JA, et al. The sSpectrum of cognitive impairment in neurocysticercosis: differences according to disease phase. Neurology 2012; 78: 861-6.

[14] Olsen RW and DeLorey TM. GABA and glycine. In: Siegel GJ, Agranoff BW, Albers RW, Fisher SK, Uhler MD, Eds. Basic Neurochemistry. New York: Lippincott-Raven 1999: pp. 335-46.

[15] Cooper E, Couturier S, Ballivet M. Pentameric structure and subunit stoichiometry of a neuronal acetylcholine receptor. Nature 1991; 350: 235-8.

[16] Saransaari P, Oja SS. Modulation of the ischemia-induced taurine release by adenosine receptors in the developing and adult mouse hippocampus. Neuroscience 2000; 97; 425-30.

[17] Castro Neto EF. Estudo da neurotransmissão aminoacidérgica no cérebro de proechimys Guyannensis guianesis submetidos ao status epiléticos induzido por pilocarpina. Master's thesis. São Paulo: UNIFESP 2003.

[18] Carpio A, Placencia M, Santillán F, Escobar A. A proposal for classification of neurocysticercosis. Can J Neurol Sci 1994; 21: 43-7.

[19] Hajek M, Do KQ, Duc C, Boesiger P and Wieser HG. Increased excitatory amino acid levels in brain cysts of epileptic patients. Epilepsy Res 1997; 28: 245-54.

[20] Wieser HG, Rudolph U, Blau N, Boison D, Imhof HG and Bernays R. Amino acid composition of brain cysts: levels of excitatory amino acids in cyst fluid fail to predict seizures. Epilepsy Res 2003; 55: 191-9.

[21] Bestetti RH. Concentração liquórica de aminoacidos neurotransmissores em pacientes epiléticos. Master's thesis. Ribeirão Preto: Faculdade de Medicina 1994.

[22] Sotelo J, Guerrero V and Rubio F. Neurocysticercosis: a new classification based on active and inactive forms. Arch Intern Med 1985; 145: 442-5.

[23] Camargo JA. Estudo dos aminoácidos neurotransmissores e da proteína Fas no líquor nas diferentes fases da neurocisticercose humana. [Tese Doutorado]. São Paulo, SP. Universidade Federal de São Paulo 2010.

[24] Camargo JA and Bertolucci PHF. Quantification of Fas protein in CSF of patients with neurocysticercosis. Arq Neuropsiquiatr 2012; 70: $264-68$. 